\title{
Transient dynamics in a nonequilibrium superdiffusive reaction-diffusion process: Nonequilibrium random search as a case study
}

\author{
F. Rusch $\odot,{ }^{1}$ M. E. Wosniack, ${ }^{2}$ E. P. Raposo, ${ }^{3,4,5}$ G. M. Viswanathan, ${ }^{6}$ and M. G. E. da Luz ${ }^{1, *}$ \\ ${ }^{1}$ Departamento de Física, Universidade Federal do Paraná, Curitiba-PR 81531-980, Brazil \\ ${ }^{2}$ Max Planck Institute for Brain Research, Frankfurt 60438, Germany \\ ${ }^{3}$ Laboratório de Física Teórica e Computacional, Departamento de Física, Universidade Federal de Pernambuco, \\ Recife-PE 50670-901, Brazil \\ ${ }^{4}$ Centre d'Estudis Avançats de Blanes-CEAB-CSIC, Girona 17300, Spain \\ ${ }^{5}$ Centre for Ecological Research and Forestry Applications-CREAF, Universitat Autònoma de Barcelona, O8193 Cerdanyola del Vallès, Spain \\ ${ }^{6}$ National Institute of Science and Technology of Complex Systems and Department of Physics, Universidade Federal do Rio Grande do \\ Norte, Natal-RN 59078-970, Brazil
}

(Received 13 March 2020; accepted 23 June 2020; published 13 July 2020)

\begin{abstract}
Transient regimes, often difficult to characterize, can be fundamental in establishing final steady states features of reaction-diffusion phenomena. This is particularly true in ecological problems. Here, through both numerical simulations and an analytic approximation, we analyze the transient of a nonequilibrium superdiffusive random search when the targets are created at a certain rate and annihilated upon encounters (a key dynamics, e.g., in biological foraging). The steady state is achieved when the number of targets stabilizes to a constant value. Our results unveil how key features of the steady state are closely associated to the particularities of the initial evolution. The searching efficiency variation in time is also obtained. It presents a rather surprising universal behavior at the asymptotic limit. These analyses shed some light into the general relevance of transients in reaction-diffusion systems.
\end{abstract}

DOI: 10.1103/PhysRevE.102.012126

\section{INTRODUCTION}

The usual approach in the study of natural processes focuses on their stationary behavior (or at steady states [1]), with much less attention being paid to the initial evolution. But transients can strongly influence a system's relaxation [2-6], determining many of its final properties $[3,7,8]$. As discussed in Ref. [3], aspects such as (a) the transient duration [6], (b) the nature of its dynamics [4,9], (c) the interconnection between the transient and the steady states characteristics [7], and (d) the state variables final values dependence on the transient $[5,7]$ can be essential to fathom statistical equilibrium.

To comprehend the long-term effects of the transient is not only a basic question. It bears many applications, such as controlling engineering systems [10] (as diverse as those based on thermal convection [11] or nuclear reactors [12]), weather prediction $[7,13]$, and understanding activation and pattern formation in biological cells $[14,15]$. As a concrete example, the above issues are relevant in ecology since the involved timescales are frequently still away from their asymptotic limits [16]. So, abrupt or strong behavioral shifts may have causation not due to environmental or the species' interactions, but due to not-yet-stabilized dynamics [17].

Reaction-diffusion processes are an important class of stochastic phenomena in which the evolution at short times can be determinant for their long run behavior $[9,14,16,18]$.

\footnotetext{
*1uz@ fisica.ufpr.br
}

This may even be enhanced if the diffusive component is anomalous, described by superdiffusive Lévy flights or walks [4,8,19-24]. For superdiffusive reaction-diffusion systems (SRDSs)—recurrent in movement ecology [25]—universal answers to the fundamental points (a)-(d) might not be possible. However, some works have pinpoint global trends in the transients of certain groups of SRDSs [21,24,26,27].

In fact, to unveil global relations between transients and steady states in a particular class of SRDS, as that associated to the foraging dynamics [23], one should seek a prototypical simple model, yet encompassing the main physical ingredients of the problems belonging to such SRDS class. A generic minimal model for the actual situation we have interest here is shown in Fig. 1. In the space $\mathcal{S}$, the number of "states" at time $t$ is $N(t)$. They are added at a given rate and can be removed through a reaction-diffusion superdiffusive mechanism. The potential different features of the individual states are not relevant since they are irreversibly annihilated whenever a reaction event takes place (but see the discussion in Sec. IV). We also note that distinct systems with absorbing states might present rather common behavior [28]. Initially, the system is out-of-equilibrium and $N(t)$ increases. But as $N(t)$ grows the annihilation probability also grows. Eventually, the steady state emerges when $N\left(t>T_{\text {stat }}\right) \approx$ const. Amazingly, certain physical aspects of biological foraging [23] can be pictured exactly as in Fig. 1.

In this contribution we elucidate the importance of the transient in establishing the steady state of a random search for targets, the "states," which are uniformly created in the 


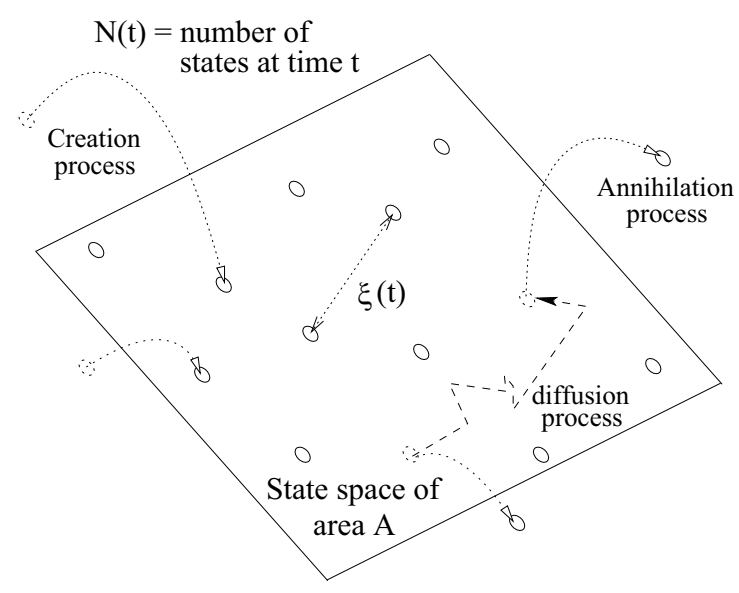

FIG. 1. Schematics of the state space $\mathcal{S}$ (of area $A$ ) in which (pertinent for the problem) "states" are randomly created at a certain rate. States annihilation is due to a stochastic reaction-diffusion process. Relevant here is the (average) number of effective states in $\mathcal{S}$ at $t, N(t)$. A typical distance in $\mathcal{S}$ can be defined by $\xi(t)=$ $\sqrt{A / N(t)}$. The transient can be characterized by a temporal variation of $N(t)$ until reaching a steady state at $t=T_{\text {stat }}$, so that $N\left(t>T_{\text {stat }}\right) \approx$ constant.

environment and are eliminated once found by a superdiffusive random walker (the searcher). The equilibrium condition arises when the targets addition and removal rates become equal.

The work is organized as the following. In Sec. II we develop our concrete model. Through numerical simulations and analytic approximations we address the previously mentioned queries (a)-(d) in Sec. III. We moreover compute the efficiency of the search process, showing its asymptotic independence on the walker diffusiveness degree. Finally, in $\mathrm{Sec}$. IV we draw our concluding remarks.

\section{THE MODEL}

The square searching arena $\mathcal{S}$, Fig. 1, has area $A$ and reflexive borders (e.g., representing a limited territory). Targets are created and annihilated in $\mathcal{S}$ through two distinct processes elaborated below. A key quantity is $N(t)$, the net number of (randomly distributed) targets in the environment at $t$. The mean spatial distance between nearby targets $\xi(t)=\sqrt{A / N(t)}$ is a concrete realization for the "typical separation" between states depicted in Fig. 1. At $t=\tau_{0}=0$, we have $N_{0}$ uniformly distributed targets.

At each $t=\tau_{n}(n=1,2,3, \ldots)$ there is an addition of $N_{n}$ targets at random positions: the creation process. Here it suffices to set $\tau_{n}=n \tau, N_{n}=1 \forall n$ (other aggregation rules are briefly discussed in Sec. IV). The targets are removed upon encounters, consequence of a random search, a reactiondiffusion dynamics [23]. If up to $t, N_{a}(t)$ is the total number of targets taken away from $\mathcal{S}$, then the targets remaining in $A$ is $N(t)=N_{c}(t)-N_{a}(t)$, with $N_{c}(t)$ the total number of targets created until $t$.

The target-depleting mechanism consists of a random walker which searches for and consumes targets as the following. It (a) chooses a random direction $0 \leqslant \theta<2 \pi$ as well as a step length $r_{0}<\ell \leqslant \sqrt{A}$ drawn from a probability density distribution $P(\ell)$. Then, (b) it starts to move with a constant speed $v$ in such direction. Along the way the walker is able to detect targets within a radius of vision $r_{0}$. If a target is spotted, then the searcher goes straight to its position and the target is removed. Otherwise, the walker finishes traveling the distance $\ell$. In both cases the whole protocol resumes, from the last stop location, with a new sorting step (a).

Different $P(\ell)$ 's will lead to distinct behavior for $N(t)$. However, a universal trend must be expected from these rules. If initially the creation is higher than the annihilation rate, then for short $t$ 's $N(t)$ increases, also increasing the chances of finding targets. Eventually these rates do equalize (at $t=T_{\text {stat }}$ ) and from then on $N\left(t>T_{\text {stat }}\right)$ become constant. Rigorously, $T_{\text {stat }}$ should be infinite. But in practice we can take $T_{\text {stat }}$ as finite by establishing that under proper conditions $N\left(t=T_{\text {stat }}\right)$ is close enough to its asymptotic. Thus, how $N$ varies with time for $t<T_{\text {stat }}$ does characterize the transient.

The above is our (exact) "discrete model" (DM) since the processes occur at particular well-defined $t$ instants. So, we will assume proper $P(\ell)$ 's and numerically simulate the evolution. But next, we also establish continuous functions of $t$ describing the problem relevant quantities. This "continuous model" (CM) can be viewed as the mean-field approximation for the system.

Obviously, $N_{c}\left(t=\tau_{n}\right)=\sum_{m=0}^{m=n} N_{m}$; thus, $N_{c}(t)=N_{0}+$ $t / \tau$. In the time interval $(t-\Delta t / 2, t+\Delta t / 2)$ (with $\Delta t$ small) we can define a mean characteristic time $\gamma(t)$ to annihilate a target, surely dependent on $\xi(t)$ and on $P(\ell)$. We further write $\gamma(\xi)=d(\xi) / v . d$ represents the average distance traveled between two successively found targets [23], being a function of $\xi$. Then, for a short $\delta t$, the probability to find a target is $p(\delta t) \sim \delta t / \gamma(t)$ and taking the limit $\delta t \rightarrow d t$, a mean-field approximation MFA (see next) for the DM reads

$$
N_{a}(t)=\int_{0}^{t} d t^{\prime} / \gamma\left[\xi\left(t^{\prime}\right)\right]
$$

From the expression for $N(t)$ and Eq. (1)

$$
d N(t) / d t=1 / \tau-1 / \gamma[\xi(t)],
$$

with

$$
\xi(t)=\sqrt{A /\left[N_{c}(t)-N_{a}(t)\right]}=\sqrt{A / N(t)} .
$$

Once one knows the functional form for $\gamma$, one can solve Eq. (2) for $N(t)$, obtaining also $N_{a}(t)$ and $\xi(t)$. The system will reach a steady condition when the number of targets in the system, $N_{\text {stat }}$ (with a corresponding $\xi_{\text {stat }}$ ), is such that the average time to eliminate a target equals to the time interval $\tau$ to add a new target. This takes place when (assuming $T_{\text {stat }}$ an appropriate finite value)

$$
\gamma\left(\xi_{\text {stat }}\right)=\tau=\gamma\left(\sqrt{A /\left[N_{c}\left(T_{\text {stat }}\right)-N_{a}\left(T_{\text {stat }}\right)\right]}\right) .
$$

For $t \geqslant T_{\text {stat }}$, Eq. (2) yields $N\left(t \geqslant T_{\text {stat }}\right)=N_{\text {stat }}$, so

$$
N_{a}\left(t \geqslant T_{\text {stat }}\right) \approx N_{a}\left(T_{\text {stat }}\right)+\left(t-T_{\text {stat }}\right) / \tau,
$$

with

$$
N_{a}\left(T_{\text {stat }}\right)=N_{0}+T_{\text {stat }} / \tau-N_{\text {stat }} .
$$


Equation (2)—with $\gamma(t)$ given next-is our "continuous" model, constituting an approximation for the DM.

Our goal is to characterize the transient until reaching stationarity for a stochastic process for which we can control its degree of diffusiveness. This can be accomplished by assuming the truncated power-law distribution $P(\ell)=P_{\mu}(\ell)=$ $C_{\mu} \ell^{-\mu}$ if $r_{0}<\ell<\sqrt{A}$ and zero otherwise, with $1<\mu \leqslant 3$ and $C_{\mu}$ the normalization constant. In the limit of $\sqrt{A}$ going to infinity, $P_{\mu}(\ell)$ represents the long-range asymptotic behavior of the family of Lévy stable distributions [19]. In this limit, for $\mu \geqslant 3$ the searching should present normal (i.e., Brownianlike) diffusion. A superdiffusive walker should correspond to $1<\mu<3$, with $\mu \rightarrow 1^{+}$displaying ballistic dynamics [23]. Also, for Lévy distributions the mean diverges if $\mu<2$, being finite (only marginally divergent) if $\mu>2(\mu=2)$. In our model, we are in fact assuming a finite $A$. However, as shown in Ref. [29] the convergence of a truncated power-law Lévy distribution to the Gaussian statistics is extremely slow. Hence, if $A$ is large enough, then for relatively long times the general properties of the nontruncated Lévy statistics (in special superdifusiveness) can be maintained [30-33] during the whole transient evolution.

For these $P_{\mu}$ 's, from the results in Refs. [34-36] for a time-independent scenario of a fixed density of targets, where $\xi=\xi_{f}$, the expression for $\gamma_{f}=\gamma\left(\lambda_{f}\right)=d_{f} / v$ is (with $\lambda_{f}=$ $\xi_{f}^{2} /\left(2 r_{0}\right)$ a "mean free path" and $\left.\mu \neq 2\right)$

$$
\gamma\left(\lambda_{f}\right)=\frac{r_{0}}{(2-\mu) v}\left[\left(\frac{\lambda_{f}}{r_{0}}\right)-(\mu-1)\left(\frac{\lambda_{f}}{r_{0}}\right)^{(\mu-1)}\right] .
$$

If $\mu=2$, then $\gamma_{f}=\lambda_{f}\left(1+\ln \left[\lambda_{f} / r_{0}\right]\right) / v$.

For our time-dependent case of $\gamma[\xi(t)]$, following the MFA for Eq. (2) we just use the above $\gamma\left(\lambda_{f}\right)$ but with $\lambda_{f} \rightarrow \lambda(t)=$ $A /\left(2 r_{0}\left[N_{c}(t)-N_{a}(t)\right]\right)$ (an "instantaneous" mean free path approximation). From Eq. (2) $N(t)$ is given by ( $\alpha=2 r_{0}^{2} / A$, $\left.\beta=\tau v / r_{0}, \mu \neq 2\right)$

$$
\int_{\alpha N_{0}}^{\alpha N(t)} d x\left(1-\frac{\beta(\mu-2) x}{\left[1+(1-\mu) x^{2-\mu}\right]+\beta(\mu-2) x}\right)=\frac{\alpha}{\tau} t .
$$

For $\mu=2$ the integrand should be $1-\beta x /(-1+\beta x+$ $\ln [x])$.

The integral in Eq. (8) can be solved for many different rational values of $1<\mu \leqslant 3$ (e.g., 3/2, 5/2, and 3 ). Thus, after integration in principle a closed expression for $N(t)$ could be derived through a proper inversion of Eq. (8), allowing to write $N$ as a function of $t$. For instance, for $\mu \rightarrow 1^{+}$, one finds

$$
N(t)=(\alpha \beta)^{-1}+\left(N_{0}-(\alpha \beta)^{-1}\right) \exp [-\alpha \beta t / \tau],
$$

which agrees with all our previous asymptotic predictions, e.g., we can fairly take [30] $T_{\text {stat }} \approx 5 N_{\text {stat }} \tau$ with $N_{\text {stat }}=$ $(\alpha \beta)^{-1}$. Note that if the integration or the subsequent inversion is not analytically possible, $N(t)$ in Eq. (8) can be easily numerically computed.

The CM is a MFA for the DM since Eq. (1) "updates" the probability to find new targets only in time [through the variation of $\gamma$ as function of $\xi(t)]$. Spatial fluctuations of $\xi$ are not considered. Recall that due to the randomness of the target creation positions, temporary spatial heterogeneities may take place, particularly at low densities. However, if the walker is effective in exploring $\mathcal{S}$, then the searching dynamics itself would mitigate the effects of such inhomogeneities. So, we expect the DM and CM to better agree for smaller $\mu$ 's, when the searcher has a stronger superdiffusive character.

Besides an approximation for the DM, the CM is also useful for another reason. Distinct phenomena [37-42] are associated to finding moving targets. It has been shown $[22,38]$ that certain dynamical aspects for fixed targets are similar to those for moving targets if one compares the former case (for a searcher of smaller $\mu$ ) with the latter (for a searcher of larger $\mu$ ) [22]. Moreover, for moving targets a MFA tends to work in a broader range of $\mu$ 's [22]. Thus, the CM might also be viewed as a simplified description of targets which, once randomly created, go through a rapid spatial homogenization or "thermalization," e.g., by diffusion.

\section{RESULTS}

We now present some relevant quantities from simulations for the DM and integration of Eq. (8) for the CM. We set $r_{0}=$ $v=1=N_{0}=1, A=10^{4} \times 10^{4}$ and take for $\tau$ the values: $\tau_{0}=2.5 \times 10^{3}, 2 \tau_{0}, 4 \tau_{0}$, and $8 \tau_{0}$. We observe that since $v=$ 1 , the numerical value of $\tau$ equals to the distance traveled by the walker during the time between two successively added targets to the environment.

Figure 2 shows $N(t)$ for the DM, CM and distinct $\tau$ 's. In Figs. 2(a)-2(d) $\mu=1.01,1.15,1.3,1.5$, values corresponding to a highly diffusive walker. For $\mu=1.1,1.5$ and $\tau=\tau_{0}$, Fig. 2(e) illustrates the small fluctuations of $N(t)$ for the DM after reaching stationarity - an absent behavior in the CM. For $\mu=2,2.5,3$ and $\tau=\tau_{0}$ (for other $\tau$ 's the results are qualitatively similar) we have the DM in Fig. 2(f) and the CM in Fig. 2(g).

From Fig. 2 we can identify few clear trends. Perhaps surprisingly, the qualitative transient dynamics for $N(t)$ is the same regardless the parameter values or model. However, $N_{\text {stat }}$ and $T_{\text {stat }}$ depend on the system details (we take $T_{\text {stat }}$ from $\left.d N(t) /\left.d t\right|_{T_{\text {stat }}} \approx 10^{-5}\right)$. The $T_{\text {stat }}$ 's are indicated by arrows in Fig. 2 for the representative cases of $\mu=1.01$ (near-ballistic) and any $\tau$; and $1.15 \leqslant \mu \leqslant 1.5$ and the lowest values of $\tau$, namely, $\tau_{0}$ and $2 \tau_{0}$. In general we observe that $N_{\text {stat }}$ and $T_{\text {stat }}$ : (i) decrease with $\tau$; (ii) increase with $\mu$ (mildly for DM if $\mu<2.0$ ); and (iii) for identical parameters, tend to be greater for CM than for DM. Last, (iv) the DM and CM have a better agreement for smaller $\mu$ 's and longer $\tau$ 's [Figs. 2(a) and 2(b)].

Feature (i) takes place because for longer $\tau$ 's the walker has more time to find/annihilate the successively added targets, more rapidly achieving the steady condition with a lower accumulation of targets at $t=T_{\text {stat }}$. As for (ii), in the present context-targets are eliminated once found-more diffusive walkers (lower $\mu$ 's) are known to be more efficient searchers [23], decreasing $T_{\text {stat }}$. This increases $N_{a}(t)$, hence it decreases $N(t)=N_{c}(t)-N_{a}(t)$ and so $N_{\text {stat }}$ (recall that $N_{c}(t)$ is a predetermined function, i.e., given by the medium). The property (iii) implies that $N_{a}(t)$ is greater for the DM than for the CM (see the explanation below). Finally, the behavior associated to (iv) corresponds to parameters for which the searcher better explores the environment. As previously mentioned [the discussion in the paragraph after Eq. (9)], a more efficient 

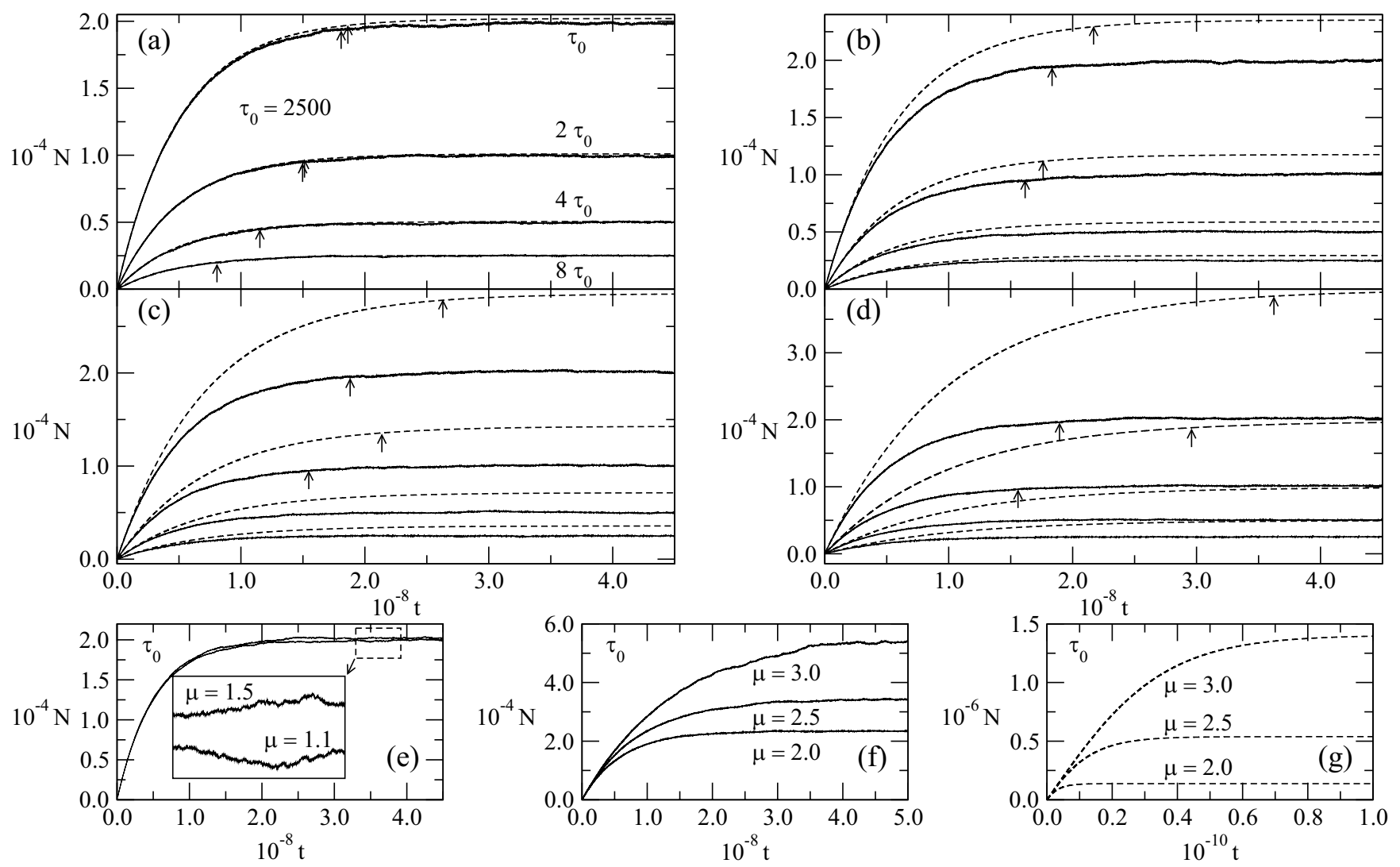

FIG. 2. The number of targets in the environment versus time, $N(t)$. The continuous (dashed) curves represent the DM (CM). For four values of $\tau$, the cases of (a) $\mu=1.01$, (b) $\mu=1.15$, (c) $\mu=1.3$, (d) $\mu=1.5$. For distinct $\mu$ 's, a same $\tau=\tau_{0}$ in panels (e)-(g). The inset in (e) illustrates the typical fluctuations in the steady state for the DM. Panels (f) and (g) contrast the magnitudes of $N_{\text {stat }}$ for the DM and CM. The small arrows in some plots indicate the corresponding $T_{\text {stat }}$.

space exploration makes the mean field a more accurate approximation.

Initially, $N(t)$ is very small and many more targets are created than annihilated. But as $N(t)$ grows, so does the rate of annihilation. This then determines the transient behavior towards the steady state, represented by $N_{a}\left(t>T_{\text {stat }}\right) \sim t$. Figure 3 shows examples of $N_{a}(t)$ for $\tau=\tau_{0}$. For other $\tau$ 's, the results are qualitatively akin, just with DM and CM tending to be closer together. When $t<T_{\text {stat }}$, Figs. 3(a)-3(c) shows that as $\mu$ increases $\left.N_{a}\right|_{\text {DM }}$ becomes systematically larger than $\left.N_{a}\right|_{\mathrm{CM}}$. To understand this, we recall that the CM assumes $\lambda$ an instantaneous mean value (over the total area $A$ ), with the average distance traveled between two targets $d$ being also an instantaneous function $d=d(\lambda(t))$. This is a less significant approximation for smaller $\mu$ 's. In the DM-due to the searching rule (b) - the walker is able to perceive local spatial fluctuations of $\lambda$ and $d$ will adjust according to the local density of targets. Thence, as one can see in Fig. 3, the search efficiency of the DM is greater than that of the CM for higher $\mu$ 's. For the DM in Fig. 3(d), $N_{a}(t)$ is depicted for some values of $1.01 \leqslant \mu \leqslant 3$ in a large range for $t$. Note that $N_{a}(t)$ increases from 0 to its maximum of $1 / \tau$ (when $\left.t \geqslant T_{\text {stat }}\right)$, characterizing the steady state.

Figures of merit $\eta$ are commonly used to classify search strategies [23]. They are ordinarily considered in stationary conditions, thus being functions solely of the system parameters. But here one should expect a time-dependent $\eta(t)$, converging to some constant value only asymptotically (say, for $t>T_{\text {stat }}^{(\eta)}>T_{\text {stat }}$ ). Among different $\eta$ 's [35,36], an often considered search efficiency [34] is the ratio between the total number of targets found and the total distance traveled $L$ up to a time $t$. Since in our idealized model it takes no time for destroying a found target and then to resume the search immediately, one gets

$$
\eta(t)=N_{a}(t) / L(t)=N_{a}(t) /(v t) .
$$

In both models one can anticipate that for $t \geqslant T_{\text {stat }}, \eta(t) \sim$ $\left[N_{a}\left(T_{\text {stat }}\right)+\left(t-T_{\text {stat }}\right) / \tau\right] /\left[L\left(T_{\text {stat }}\right)+v\left(t-T_{\text {stat }}\right)\right]$, thus

$$
\eta(t \rightarrow \infty)=1 /(v \tau)
$$

contrasting with many random search instances where $\eta=\eta(\mu)$ [23]. Moreover, we have that $T_{\text {stat }}^{(\eta)} \gg T_{\text {stat }}$ and $\eta(t) /(v \tau)^{-1} \approx p$ implies $t \approx \tau N_{\text {stat }} /(1-p)$. For example, for $\eta(t)$ to be $99 \%$ of its maximum, $t \sim 100 \times T_{\text {stat }}$. However, a better way to infer (say, from empirical data [41]) the efficiency asymptotic value is by calculating the rate between the found targets and the distance traveled by the searcher in a given time interval, or

$$
d N_{a}(t) / d L=v^{-1} d N_{a}(t) / d t .
$$

From such computation we obtain $d N_{a}(t) / d L \approx(v \tau)^{-1}$ already for $t \gtrsim T_{\text {stat }}$ (with $T_{\text {stat }}$ the $N(t)$ stationary time of the corresponding model). 

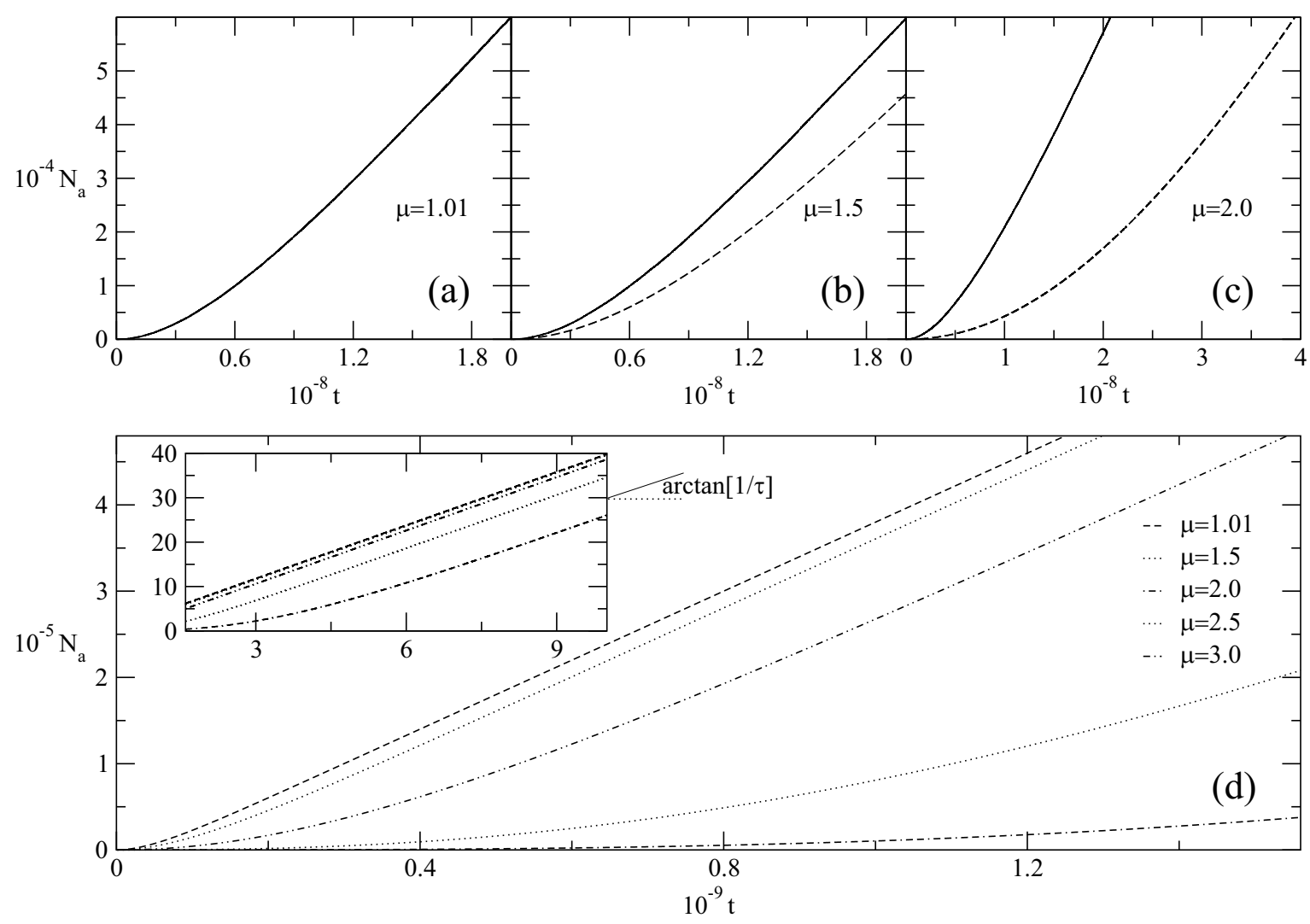

FIG. 3. The total number of found targets versus time, $N_{a}(t)$, for distinct $\mu$ 's and $\tau=\tau_{0}=2.5 \times 10^{3}$. (a-c) The continuous (dashed) lines represent the DM (CM) prior to reach the steady state. (d) For the DM, the $N_{a}(t)$ behavior for different $\mu$ 's. The inset shows times close to $T_{\text {stat }}$ (after which $N_{a}(t)$ must be a straight line of angular coefficient $\tan [1 / \tau]$ ).

For longer $\tau$ 's, the DM and CM give closer results, Fig. 2. Also, the overall shape of $N(t)$ does not change with $\tau$. So, in Fig. 4 we display $\eta(t)$ only for $\tau=\tau_{0}$. For small $\mu$ 's the $\mathrm{DM}$ and $\mathrm{CM}$ are similar, leading to a reasonable agreement between the respective $\eta(t)$ 's as seen in Figs. 4(a) and 4(b). For $\mu \geqslant 2.0$ in Figs. 4(c)-4(e), the increasing of $\eta$ with $t$ is faster for the DM than for the CM. For the CM, Figs. 4(a)-4(e) shows that $d N_{a}(t) / d L$ tends more rapidly to the asymptotics $(v \tau)^{-1}$ than the usual efficiency function $\eta(t)$. Finally, for the DM and distinct $\mu$ 's, Fig. 4(f) illustrates that it takes a very long time $T_{\text {stat }}^{(\eta)}$ for $\eta(t)$ to achieve its asymptotic value.

\section{FINAL REMARKS AND CONCLUSION}

As previously mentioned in the Introduction, transient dynamics may be fundamental in tailoring the characteristics of the steady state [4-6], thus determining the system asymptotic features $[10,21,43]$. But in many instances, especially in ecology [44-46], to properly establish these links is not a simple task $[47,48]$. Random search in general and biological foraging in particular, important examples of SRDS, are not exceptions to this difficulty $[23,25]$. For example, it is well known that animals behavior can mold their landscape through feedback processes [49]. But unfortunately, to predict the environmental potential changes from these mutual interactions is still far from being a settled problem [50,51].

In this work we have investigated a simple, nonetheless illustrative, example of a superdiffusive random search in a nonequilibrium "medium." We have shown that the properties at the steady state are clearly associated to the transient period. Indeed, many quantities characterizing the system after reaching the stationarity for $N(t)$ are clearly functions of the early stages of the evolution. More concretely, we have addressed [3]: (a) the transient duration, (b) its dynamical characteristics, (c) the relation between the transient and the final steady states, and (d) the state variables ultimate values dependence on the transient. For so we have performed exact simulations (of course, up to the computational numerical precision but absolutely not an issue for our model straightforward rules) and a mean-field analytic approximation. By comparing their predictions, we could determine for which range of parameters mean-field methods are or are not reliable in describing this type of problem. Also, there are very few analytical approaches for random search models [22] and this can be considered an extra contribution to the area.

A result obtained here, contrasting with most of the random search models [23,25], is that the efficiency $\eta(t)$, although having a $\mu$-dependent slop (see Fig. 4), converges to an asymptotic value which is independent on $\mu$. This is due to a sort of interactive correlation between the searcher and the landscape. Commonly (but see Refs. $[22,38,40]$ ), unless for very local fluctuations $[35,36]$ the environment is assumed static and the searching efficiency is basically determined by the searcher features, e.g., its diffusiveness (specified by $\mu$ ). In the present case, $\mu$ is associated to the rate in which the targets are found, explaining the $\eta(t)$ relation with $\mu$. However, in 

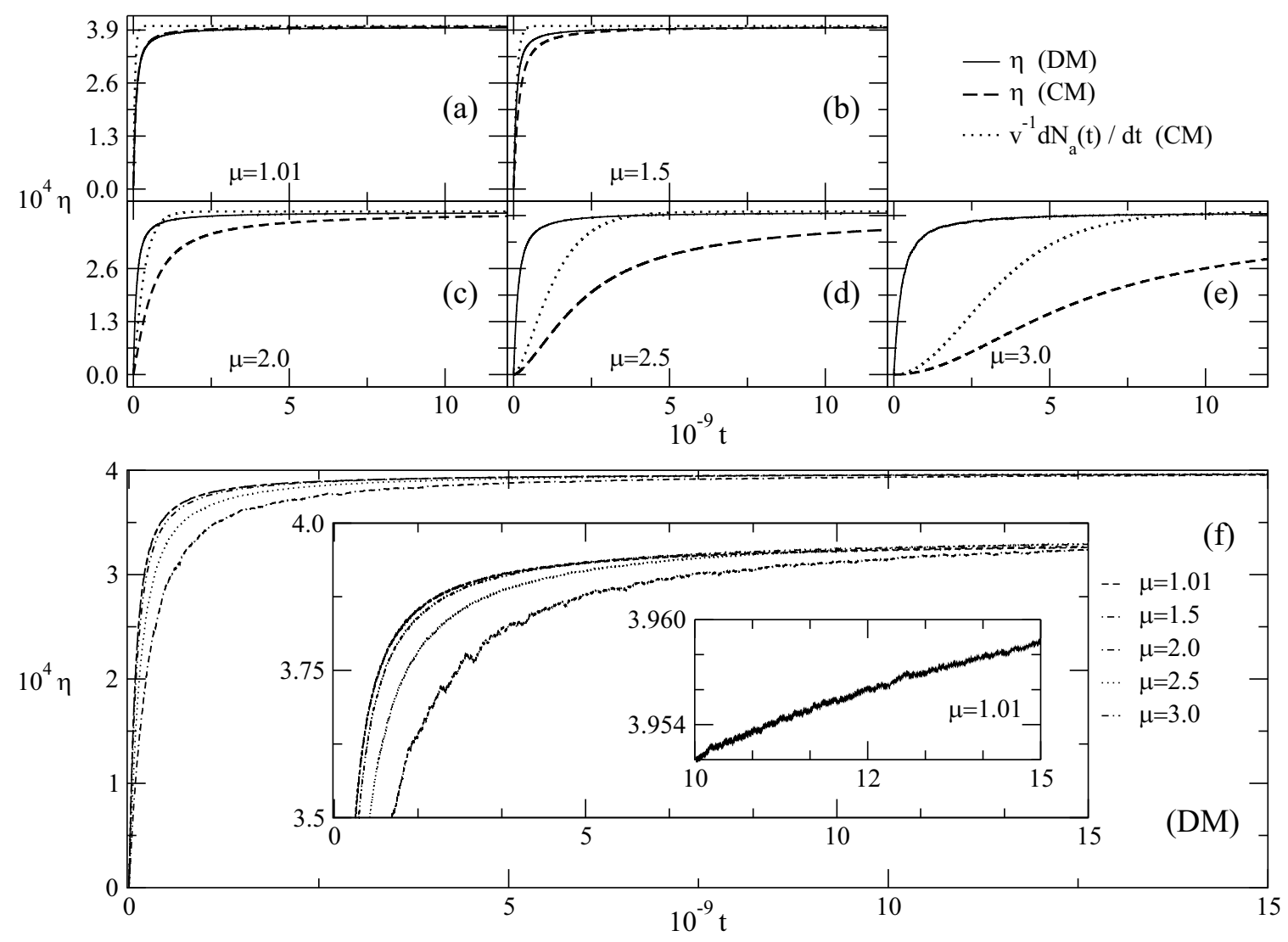

FIG. 4. The efficiency $\eta(t)$ for different $\mu$ 's and $\tau=\tau_{0}=2.5 \times 10^{3}$. (a-e) Comparison between the results for the DM and CM. The quantity $d N_{a} / d L=v^{-1} d N_{a}(t) / d t$ for the CM is also shown. (f) For the DM, $\eta(t)$ for distinct values of $\mu$. Blow ups at different timescales illustrate the long time necessary for $\eta(t)$ to tend to its limit value.

the asymptotic limit the searcher will deplete the medium at the same pace in which new targets are supplied to it (at the frequency $1 / \tau$, an exclusive environment property). Since the efficiency is defined as the number of targets found by the distance traveled, at the steady state this is equivalent to $\eta=$ $1 / d$ [23] [we recall that $d$ is the distance traveled between two found targets and asymptotically $d=d\left(\xi_{\text {stat }}\right)=$ constant]. At the steady state $d / v=\tau_{d}=\tau$ regardless of $\mu$, elucidating our findings.

Obviously, this is a first minimal model and many other aspects could be added to the system description. Next we shall comment on just two possibilities. We have considered all targets being similar. But we could assume families, $i=$ $1,2,3, \ldots$, of targets, each with its own creation time $\tau_{i}$. It has been shown that if the gain per encounter is different depending on the type of target found, the efficiency becomes a rather involving function, depending on distinct factors of the search dynamics and landscape configuration [52]. Certainly, this should strongly influence the transient as well as $\eta(t)$ and its asymptotic limit value.

Second, for our single type of target we have assumed a constant $\tau$ yielding a linear $N_{c}(t)$. However, one can imagine diverse target creation mechanisms leading to various $N_{c}(t)$ 's. Of course, if at each time interval $\tau$ a constant number of targets $N_{\tau}$ is added to the system, the general phenomenology would be basically the same, just with the steady state being achieved when $\tau_{d}=\tau / N_{\tau}$ (see above). The situation is far more richer if $N_{\tau}$ is not constant. For example, an ever increasing $N_{\tau}$ may represent an interesting mathematical model, but probably unrealistic for describing a typical searching problem. In this case $N(t)$ cannot become stationary. However, a particularly interesting context is for an oscillating $N_{\tau}$, eventually representing seasonal variations of an ecosystem [49]. This would result $N(t)$ also oscillatory, potentially displaying out-of-phase and delay behavior [43]. Such effects will be the subject of a forthcoming contribution.

To conclude, concerning the important physical points (a), (c), and (d) connecting the transient to the steady state, we have found that certain global characteristics (e.g., diffusiveness and targets creation rate) determine features of the initial evolution. In turn, the transient establishes the final stationary environment configuration (e.g., the "population" $N_{\text {stat }}$ ), with a $\mu$-independent $\eta(t \rightarrow \infty)$. As for (b), irrespective of the parameters or description details (DM or CM), the qualitative transient dynamics (say, of $N$ and $N_{a}$ ) is universal. All these results closely agree with the view of landscape ecology as an emergent complex system [53] (common for SRDSs $[5,6])$. The novelty is that in the present case it is driven by transients. In fact, our study adds to the list of ecological systems where transient regimes play a fundamental role $[16,17,48]$. 


\section{ACKNOWLEDGMENTS}

This work was supported by Brazilian agencies $\mathrm{CNPq}$, CAPES, and FACEPE. M.E.W. thanks a Capes-Humboldt postdoctoral fellowship. We are in great debt to L. Giuggioli for fruitful discussions in the very early stage of this study. Finally, we thank the anonymous referees for valuable comments helping us to improve the discussions in the manuscript.
[1] One finds close although distinct definitions of a "steady state" in the literature. Here it means the system may present a time dynamics, but with relevant quantities remaining constant if averaged in short time intervals.

[2] C. D. Alstad, H. S. Isbin, N. R. Amudson, and J. P. Silvers, AIChE 1, 417 (1995).

[3] S. Panchev, CR. Acad. Bulg. Sci. 56, 37 (2003).

[4] J. Spiechowicz, J. Luczka, and P. Hänggi, Sci. Rep. 6, 30948 (2016).

[5] J. Grela, Phys. Rev. E 96, 022316 (2017).

[6] T. Kittel, J. Heitzig, K. Webster, and J. Kurths, N. J. Phys. 19, 083005 (2017).

[7] S. Kravtsov, N. Sugiyama, and A. A. Tsoni, Nonlin. Processes Geophys. 1, 1905 (2014).

[8] V. Latora, A. Rapisarda, and S. Ruffo, Phys. Rev. Lett. 83, 2104 (1999).

[9] S. Ishihara, M. Otsuji, and A. Mochizuki, Phys. Rev. E 75, 015203(R) (2007).

[10] S. A. Marshal, in Steady-state and transient behavior of control systems, Introduction to Control Theory (Palgrave, London, 1978), pp. 73-104.

[11] G. Dai and J. Huang, J. Appl. Phys. 124, 235103 (2018).

[12] B.-Il Jang, M. W. Kim and G. Jeun, Nucl. Technol. 177, 203 (2012).

[13] V. Glenis, V. Pinamonti, J. W. Hall, and C. G. Kilsby, Adv. Water Resour. 85, 14 (2015).

[14] I. Hecht, D. A. Kessler, and H. Levine, Phys. Rev. Lett. 104, 158301 (2010).

[15] G. Campagnola, K. Nepal, B. W. Schroder, O. B. Peersen, and D. Krapf, Sci. Rep. 5, 17721 (2015).

[16] A. Hastings, Trends Ecol. Evol. 19, 39 (2004).

[17] A. Hastings et al., Science 361, eaat6412 (2018).

[18] A. Wacker, S. Bose, and E. Schöll, Europhys. Lett. 31, 257 (1995).

[19] Lévy Flights and Related Topics in Physics, edited by M. F. Shlesinger, G. Zaslavsky, U. Frisch (Springer, Berlin, 1995).

[20] G. Zumofen, J. Klafter, and M. F. Shlesinger, Phys. Rev. Lett. 77, 2830 (1996).

[21] D. Brockmann and L. Hufnagel, Phys. Rev. Lett. 98, 178301 (2007).

[22] C. L. Faustino, G. M. Viswanathan, M. G. E. da Luz, E. P. Raposo, and L. R. Silva, Europhys. Lett. 77, 30002 (2007).

[23] G. M. Viswanathan, M. G. E. da Luz, E. P. Raposo, and H. E. Stanley, The Physics of Foraging: An Introduction to Random Searches and Biological Encounters (Cambridge University Press, Cambridge, UK, 2011).

[24] S. Carnaffan and R. Kawai, Phys. Rev. E 99, 062120 (2019).

[25] V. Méndez, D. Campos, and F. Bartumeus, Stochastic Foundations in Moviment Ecology (Springer, Berlin, 2014).

[26] D. Applebaum, Not. Am. Math. Soc. 51, 1336 (2004).

[27] N. Sandrić, Stochastics 88, 1012 (2016).
[28] M. M. de Oliveira, M. G. E. da Luz, and C. E. Fiore, Phys. Rev. E 92, 062126 (2015).

[29] R. N. Mantegna and H. E. Stanley, Phys. Rev. Lett. 73, 2946 (1994).

[30] G. M. Viswanathan, E. P. Raposo, F. Bartumeus, J. Catalan, and M. G. E. da Luz, Phys. Rev. E 72, 011111 (2005).

[31] F. Bartumeus, J. Catalan, G. M. Viswanathan, E. P. Raposo, and M. G. E. da Luz, J. Theor. Biol. 252, 43 (2008).

[32] S. A. Sotelo-López, M. C. Santos, E. P. Raposo, G. M. Viswanathan, and M. G. E. da Luz, Phys. Rev. E 86, 031133 (2012).

[33] M. E. Wosniack, M. C. Santos, E. P. Raposo, G. M. Viswanathan, and M. G. E. da Luz, Phys. Rev. E 91, 052119 (2015).

[34] G. M. Viswanathan, S. V. Buldyrev, S. Havlin, M. G. E. da Luz, E. P. Raposo, and H. E. Stanley, Nature 401, 911 (1999).

[35] E. P. Raposo, S. V. Buldyrev, M. G. E. da Luz, M. C. Santos, H. E. Stanley, and G. M. Viswanathan, Phys. Rev. Lett. 91, 240601 (2003).

[36] M. C. Santos, E. P. Raposo, G. M. Viswanathan, and M. G. E. da Luz, Europhys. Lett. 67, 734 (2004).

[37] R. M. R. Barclay and R. M. Brigham, Anim. Behav. 48, 1013 (1994).

[38] F. Bartumeus, P. Fernández, M. G. E. da Luz, J. Catalan, R. V. Solé, and S. A. Levin, Eur. Phys. J. Spec. Topics 157, 157 (2008).

[39] G. Hollinger, S. Singh, J. Djugash, and A. Kehagias, Int. J. Robot. Reser. 28, 201 (2009).

[40] C. L. Faustino, M. L. Lyra, E. P. Raposo, G. M. Viswanathan, and M. G. E. da Luz, Europhys. Lett. 97, 50005 (2012).

[41] E. Kagan and I. B.-Gal, Search and Foraging: Individual Motion and Swarm Dynamics (CRC Press, Boca Raton, FL, 2015).

[42] J. A. Nunez, D. I. Singham, and M. P. Atkinson, J. Operat. Reser. Soc. 71, 589 (2020).

[43] Y.-C. Lai and T. Tél, Transient Chaos: Complex Dynamics on Finite Timescales (Springer, Berlin, 2011).

[44] M. F. Shlesinger and J. Klafter, in On Growth and Form, edited by H. E. Stanley and N. Ostrowsky (Nijhoff, Dordrecht, 1986), p. 279.

[45] T. M. Massie, A. Ryabov, B. Blasius, G. Withoff, and U. Gaedke, Am. Nat. 182, 103 (2013).

[46] F. Caravelli and P. P. A. Staniczenko, Plos One 11, e0157876 (2016).

[47] D. L. DeAngelis and J. C. Waterhouse, Ecol. Monogr. 57, 1 (1987).

[48] A. Morozov, K. Abbott, K. Cuddington, T. Francis, G. Gellner, A. Hastings, Y.-C. Lai, S. Petrovskii, K. Scranton, and M. L. Zeeman, Phys. Life Rev. 32, 1 (2020).

[49] F.-K. Holtmeier, Animals' Influence on the Landscape and Ecological Importance (Springer, Dordrecht, 2015). 
[50] P. M. Vitousek, H. A. Mooney, J. Lubchenco, and J. M. Melillo, Science 277, 494 (1997).

[51] A. M. Milner, C. L. Fastie, F. S. Chapin III, D. R. Engstrom, and L. C. Sharman, BioScience 57, 237 (2007).
[52] M. E. Wosniack, E. P. Raposo, G. M. Viswanathan, and M. G. E. da Luz, Phys. Rev. E 92, 062135 (2015).

[53] E. A. Newman, M. C. Kennedy, D. A. Falk, and D. McKenzie, Front. Ecol. Evol. 7, 293 (2019). 